University of Wollongong

Research Online

Australian Institute for Innovative Materials -

Papers

Australian Institute for Innovative Materials

$1-1-2018$

Complete stress-induced depolarization of relaxor ferroelectric crystals without transition through a non-polar phase

Sergey Shkuratov

Loki Incorporated

Jason Baird

Missouri University of Science and Technology

Vladimir Antipov

Loki Incorporated

Wesley Hackenberger

Trs Technologies, Inc.

Jun Luo

Trs Technologies, Inc

See next page for additional authors

Follow this and additional works at: https://ro.uow.edu.au/aiimpapers

Part of the Engineering Commons, and the Physical Sciences and Mathematics Commons

Research Online is the open access institutional repository for the University of Wollongong. For further information contact the UOW Library: research-pubs@uow.edu.au 


\title{
Complete stress-induced depolarization of relaxor ferroelectric crystals without transition through a non-polar phase
}

\author{
Abstract \\ The development of relaxor ferroelectric single crystal technology is driven by the ability to tailor \\ ferroelectric properties through domain engineering not achievable in polycrystalline materials. In this \\ study, three types of domain-engineered rhombohedral $\mathrm{Pb}(\operatorname{In~1/2~Nb~1/2)0~} 3-\mathrm{Pb}(\mathrm{Mg} 1 / 3 \mathrm{Nb} 2 / 3) 03$ \\ -PbTiO 3 crystals were subjected to transverse high strain rate loading. The experimental results indicate \\ that the domain configuration has a significant effect on the stress-induced depolarization and the \\ associated charge released. A complete depolarization of the single-domain crystals with $3 \mathrm{~m}$ symmetry \\ is observed, while multidomain crystals with $4 \mathrm{~mm}$ and $\mathrm{mm} 2$ symmetries retain a fraction of their initial \\ remanent polarization. The complete depolarization of single-domain crystals is unique without transition \\ to a non-polar phase, with a stress-induced charge density of $0.48 \mathrm{C} / \mathrm{m} 2$. This is up to three times higher \\ than that of the multidomain crystals and $\mathrm{PbZr} \times \mathrm{Ti} 1-\mathrm{x} 03$ ferroelectric ceramics that are critical for \\ ultrahigh-power transducer applications. The main offering of this work is to propose a detailed \\ mechanism for complete stress-induced depolarization in ferroelectric crystals which doe s not involve an \\ intermediate transformation to a non-polar phase.

\section{Disciplines} \\ Engineering | Physical Sciences and Mathematics

\section{Publication Details} \\ Shkuratov, S. I., Baird, J., Antipov, V. G., Hackenberger, W., Luo, J., Zhang, S., Lynch, C. S., Chase, J. B., Jo, H. \\ R. \& Roberts, C. C. (2018). Complete stress-induced depolarization of relaxor ferroelectric crystals without \\ transition through a non-polar phase. Applied Physics Letters, 112 (12), 122903-1-122903-5.

\section{Authors} \\ Sergey Shkuratov, Jason Baird, Vladimir Antipov, Wesley Hackenberger, Jun Luo, Shujun Zhang, C S. Lynch, \\ Jay Chase, Hwan Jo, and Christopher C. Roberts
}




\section{Complete stress-induced depolarization of relaxor ferroelectric crystals without transition through a non-polar phase}

Sergey I. Shkuratov, Jason Baird, Vladimir G. Antipov, Wesley Hackenberger, Jun Luo, Shujun Zhang, Christopher S. Lynch, Jay B. Chase, Hwan R. Jo, and Christopher C. Roberts

Citation: Appl. Phys. Lett. 112, 122903 (2018); doi: 10.1063/1.5019593

View online: https://doi.org/10.1063/1.5019593

View Table of Contents: http://aip.scitation.org/toc/apl/112/12

Published by the American Institute of Physics

\section{Articles you may be interested in}

Nanoscale insight of high piezoelectricity in high-TC PMN-PH-PT ceramics

Applied Physics Letters 112, 122902 (2018); 10.1063/1.5022105

Enhanced electrocaloric effect in La-based PZT antiferroelectric ceramics

Applied Physics Letters 112, 122904 (2018); 10.1063/1.5018431

Field-induced phase transitions and enhanced double negative electrocaloric effects in ( $\mathrm{Pb}, \mathrm{La})(\mathrm{Zr}, \mathrm{Sn}, \mathrm{Ti}) \mathrm{O}_{3}$ antiferroelectric single crystal

Applied Physics Letters 112, 133901 (2018); 10.1063/1.5018790

Stabilized antiferroelectricity in $\mathrm{xBiScO}_{3}-(1-\mathrm{x}) \mathrm{NaNbO}_{3}$ lead-free ceramics with established double hysteresis loops

Applied Physics Letters 112, 092905 (2018); 10.1063/1.5017697

Enhanced energy storage density by inducing defect dipoles in lead free relaxor ferroelectric $\mathrm{BaTiO}_{3}$-based ceramics

Applied Physics Letters 110, 132902 (2017); 10.1063/1.4979467

Gradient chemical order in the relaxor $\mathrm{Pb}\left(\mathrm{Mg}_{1 / 3} \mathrm{Nb}_{2 / 3}\right) \mathrm{O}_{3}$

Applied Physics Letters 112, 082901 (2018); 10.1063/1.5016561

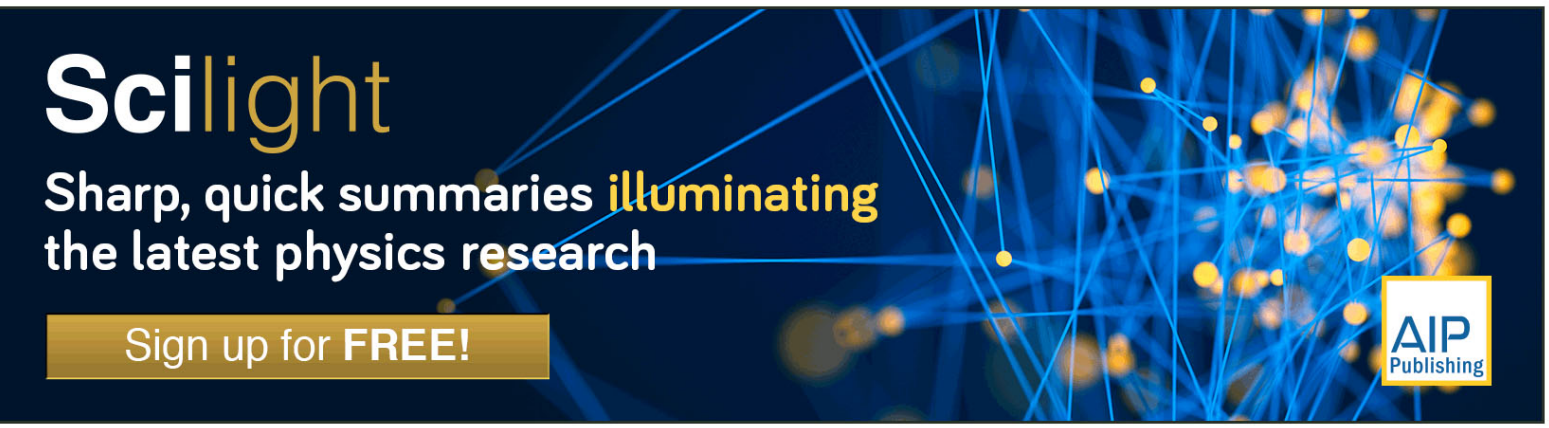




\title{
Complete stress-induced depolarization of relaxor ferroelectric crystals without transition through a non-polar phase
}

\author{
Sergey I. Shkuratov, ${ }^{1, a)}$ Jason Baird, ${ }^{1,2}$ Vladimir G. Antipov, ${ }^{1}$ Wesley Hackenberger, ${ }^{3}$ \\ Jun Luo, ${ }^{3}$ Shujun Zhang, ${ }^{4}$ Christopher S. Lynch, ${ }^{5}$ Jay B. Chase, ${ }^{1}$ Hwan R. Jo, ${ }^{5}$ \\ and Christopher C. Roberts ${ }^{5}$ \\ ${ }^{1}$ Loki Incorporated, Norwood, Missouri 65717, USA \\ ${ }^{2}$ Department of Mining and Nuclear Engineering, Missouri University of Science and Technology, Rolla, \\ Missouri 65409-0450, USA \\ ${ }^{3}$ TRS Technologies Incorporated, State College, Pennsylvania 16801, USA \\ ${ }^{4}$ ISEM, AIIM, University of Wollongong, Wollongong, NSW 2500, Australia \\ ${ }^{5}$ Department of Mechanical and Aerospace Engineering, University of California at Los Angeles, Los Angeles, \\ California 90095-1597, USA
}

(Received 14 December 2017; accepted 10 March 2018; published online 20 March 2018)

\begin{abstract}
The development of relaxor ferroelectric single crystal technology is driven by the ability to tailor ferroelectric properties through domain engineering not achievable in polycrystalline materials. In this study, three types of domain-engineered rhombohedral $\mathrm{Pb}\left(\mathrm{In}_{1 / 2} \mathrm{Nb}_{1 / 2}\right) \mathrm{O}_{3}-\mathrm{Pb}\left(\mathrm{Mg}_{1 / 3} \mathrm{Nb}_{2 / 3}\right) \mathrm{O}_{3}-\mathrm{PbTiO}_{3}$ crystals were subjected to transverse high strain rate loading. The experimental results indicate that the domain configuration has a significant effect on the stress-induced depolarization and the associated charge released. A complete depolarization of the single-domain crystals with $3 m$ symmetry is observed, while multidomain crystals with $4 \mathrm{~mm}$ and $\mathrm{mm} 2$ symmetries retain a fraction of their initial remanent polarization. The complete depolarization of single-domain crystals is unique without transition to a non-polar phase, with a stress-induced charge density of $0.48 \mathrm{C} / \mathrm{m}^{2}$. This is up to three times higher than that of the multidomain crystals and $\mathrm{PbZr}_{\mathrm{x}} \mathrm{Ti}_{1-\mathrm{x}} \mathrm{O}_{3}$ ferroelectric ceramics that are critical for ultrahigh-power transducer applications. The main offering of this work is to propose a detailed mechanism for complete stress-induced depolarization in ferroelectric crystals which does not involve an intermediate transformation to a non-polar phase. Published by AIP Publishing.
\end{abstract}

https://doi.org/10.1063/1.5019593

Relaxor based ferroelectric (FE) single crystals such as $\mathrm{Pb}\left(\mathrm{In}_{1 / 2} \mathrm{Nb}_{1 / 2}\right) \mathrm{O}_{3}-\mathrm{Pb}\left(\mathrm{Mg}_{1 / 3} \mathrm{Nb}_{2 / 3}\right) \mathrm{O}_{3}-\mathrm{PbTiO}_{3}$ (PIN-PMN-PT) have been actively studied due to their exceptional electromechanical properties. ${ }^{1-7}$ This has led to the development of a new generation of piezoelectric sensors and imaging transducers with improved performance. Recent research has focused on achieving an increase in power from the hundred milliwatts to kilowatt level for high-power resonant transducers used in therapeutic applications. ${ }^{89}$ A further increase in power to the megawatt level can be achieved with shock loading. This enables their use in autonomous pulsed power microwave generator applications. ${ }^{10}$ The operation of ferroelectric crystals in the low-strain mode is reliable, while the operation in the high-power mode is limited by stress and electric field induced phase transitions, loss of remanent polarization, and damage to the crystals. Although this degradation of piezoelectric properties can limit their application in high-power systems, rapid depolarization under shock loading can result in the generation of megawatt power levels for a brief interval of time. Ultrahigh-power ferroelectric systems based on shock compression of $\mathrm{PbZr}_{\mathrm{x}} \mathrm{Ti}_{1-\mathrm{x}} \mathrm{O}_{3}$ (PZT) ferroelectrics are capable of producing pulses of kiloamperes of current and hundreds of kilovolts of electric potential. ${ }^{10,11}$ However, imperfections in the crystal structure, which ranges from voids and inclusions to the uncontrollable crystallographic orientation of the grains in PZT ceramics, ${ }^{12}$ limit the power that can be generated in ultrahigh-power applications. Studies of the depolarization mechanisms in crystals under high stress are

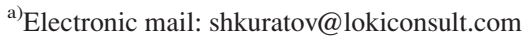

important for the identification of possible limitations in the high-power mode and to the successful development of highpower and ultrahigh-power transducers.

The ferroelectric and piezoelectric properties of crystals can be enhanced through domain engineering, i.e. creating different domain configurations by polarizing crystals in specific directions relative to the crystallographic orientation. Specific orientations can be selected where, once poled, there is no driving force for domain wall motion under a positive electric field. This results in enhanced electro-mechanical coupling with a corresponding low dielectric loss and extraordinarily large piezoelectric coefficients. The resulting macroscopic (volume average) polarization symmetry is associated with the engineered dipole alignment of the rhombohedral domains. In this paper, the results are reported from experimental studies of the remanent polarization change in PIN-PMN-PT crystals with different macroscopic domain pattern symmetries subjected to high strain rate loading. The important finding is that the domain-engineered state plays an important role in the degree of stress-induced depolarization. This is evident from the measured change of electric charge density where [111]c poled PIN-PMN-PT single-domain crystals were completely depolarized under 3.9 GPa stress and released a charge density equal to their initial remanent polarization, $0.48 \mathrm{C} / \mathrm{m}^{2}$, which is $250 \%$ and $150 \%$ higher than that for [001]c and [011]c poled multidomain crystals, respectively.

The mechanism of the complete depolarization of singledomain crystals is inferred by considering the known depolarization mechanisms in ferroelectrics. Ferroelectrics can be 
completely depolarized through a phase transformation to a non-polar cubic phase by heating above the Curie temperature or by the application of tens of gigapascals compressive stress. ${ }^{13}$ The exception is $\mathrm{PbZr}_{0.95} \mathrm{Ti}_{0.05} \mathrm{O}_{3}$ doped $2 \% \mathrm{Nb}$ (PZT 95/5) developed by Sandia National Laboratories in the 1960s. PZT 95/5 can be completely depolarized when driven through a ferroelectric to antiferroelectric phase transition induced by relatively low amplitude $(0.25-0.30 \mathrm{GPa})$ hydrostatic compression. ${ }^{14}$ The stress-induced complete depolarization of singledomain crystals reported here is unique in that it appears to occur without a transition to a non-polar phase, but it is the result of a shock-induced ferroelectric to ferroelectric phase transformation and polarization reorientation driven by release waves propagating behind the shock front. The obtained results contribute to knowledge of fundamental properties of ferroelectric materials and suggest promising applications of domainengineered crystals.

PIN-PMN-PT single crystals were grown by TRS Technologies, Inc. using the modified Bridgman technique. ${ }^{3,15,16}$ All crystal specimens were selected to be in the ferroelectric rhombohedral phase and compositionally in close proximity to the morphotropic phase boundary, $0.27 \mathrm{~Pb}\left(\mathrm{In}_{1 / 2}\right.$ $\left.\mathrm{Nb}_{1 / 2}\right) \mathrm{O}_{3}-0.47 \mathrm{~Pb}\left(\mathrm{Mg}_{1 / 3} \mathrm{Nb}_{2 / 3}\right) \mathrm{O}_{3}-0.3 \mathrm{PbTiO}_{3}$. Each PIN-PMNPT crystal specimen was measured 5.0-mm long $\times 5.0-\mathrm{mm}$ wide $\times 5.0-\mathrm{mm}$ thick. The complete set of material constants was determined by combined resonance and ultrasonic methods. Experimental details are described elsewhere. ${ }^{17}$ Physical properties of PIN-PMN-PT crystals are listed in Table I. Typical polarization hysteresis loops are presented in Fig. SM1 (supplementary material).

Poled rhombohedral PIN-PMN-PT crystals were shockcompressed perpendicular to the polarization (transverse shock). The loading arrangement utilizes a high explosive (HE) shock compression scheme. Schematics of the experimental device used for high strain rate loading studies and the measuring circuits are shown in Fig. SM-2 (supplementary material). Additional experimental details are described elsewhere. ${ }^{11,18}$

For rhombohedral crystals, poling along the [001]c and [011]c directions induces engineered multidomain configurations with $4 \mathrm{~mm}$ and $\mathrm{mm} 2$ macroscopic symmetries, respectively. The poling along the [111]c spontaneous polarization direction induces $3 m$ symmetry with a single-domain state.

When shock loaded, the high amplitude stress wave that propagates across the ferroelectric crystals produces a current versus time profile. The stress-induced electric charge released is the time integral of the stress-induced current. Typical waveforms of the stress-induced current and dynamics of electric charge density released by multidomain crystals with $4 m m$ and $m m 2$ symmetries are shown in Figs. 1(a) and 1(b). The crystals of both types were shock-compressed along the [100]c direction. The insets show typical stress-induced current waveforms for PZT 95/5 and $\mathrm{PbZr}_{0.52} \mathrm{Ti}_{0.48} \mathrm{O}_{3}$ (PZT 52/ 48) ceramic specimens used as references.

Figures 1(c) and 1(d) present typical waveforms of stress-induced current and dynamics of electric charge density released by single-domain crystals with $3 m$ symmetry. Crystals were shocked along two transverse directions, $[1 \overline{1} 0] \mathrm{c}$ and $[11 \overline{2}] \mathrm{c}$. Experimental results indicate that there is no difference in stress-induced charge density released by these crystals.

The direction of stress-induced current provides information about the origin of electric charge generated by ferroelectrics under high stress. The direction of the stress-induced current produced by crystals with the three domain configurations was identical to that for PZT ceramics (Fig. 1). Under transverse shock compression, the linear piezoelectric effect would cause a current in the direction opposite to what was observed in the experiments. This indicates that the charge released by the crystals was caused not by the piezoelectric effect but by other factors that led to depolarization. Similar to PZT ceramics, the rhombohedral PIN-PMN-PT crystals were depolarized under high strain rate loading.

The experimental results for PIN-PMN-PT crystals along with PZT ceramics are summarized in Table I. The obtained results (Fig. 1 and Table I) indicate that the initial engineered domain state has a significant effect on the waveforms of stress-induced current and charge densities generated by the crystals. The single-domain crystals with $3 m$ symmetry were completely depolarized under high stress and released charge density $0.48 \mathrm{C} / \mathrm{m}^{2}$, which is $250 \%$ and $150 \%$ higher than that for multidomain crystals with $4 \mathrm{~mm}$ and $\mathrm{mm} 2$ symmetries, respectively. Understanding the mechanism responsible for the stress-induced complete depolarization of single-domain crystals is important for the development of a new class of materials for ultrahigh-power transducers.

Polarization reorientation can occur when the component of stress parallel to the polarization direction is more compressive than that perpendicular to the polarization direction. Thus, uniaxial transverse adiabatic compression would not be expected to induce immediate rotation of the polarization. The hydrostatic compression component of the stress tensor, however, can induce structural transformations with an associated volume reduction, resulting in depolarization of the ferroelectrics. The

TABLE I. Experimentally obtained stress-induced charge densities and physical properties of rhombohedral PIN-PMN-PT crystals and PZT 52/48 and PZT $95 / 5$ ceramic specimens.

\begin{tabular}{|c|c|c|c|c|c|}
\hline Property & PIN-PMN-PT [001]c cut & PIN-PMN-PT [011]c cut & PIN-PMN-PT [111]c cut & PZT 52/48 & PZT 95/5 \\
\hline Domain pattern symmetry & $4 m m$ & $m m 2$ & $3 m$ & $\infty \mathrm{m}$ & $\infty \mathrm{m}$ \\
\hline Density $\left(10^{3} \mathrm{~kg} / \mathrm{m}^{3}\right)$ & 8.1 & 8.1 & 8.1 & 7.5 & 7.9 \\
\hline Dielectric constant ${ }^{\mathrm{a}}$ & 6700 & 5100 & 1180 & 1300 & 295 \\
\hline Elastic constant $\mathrm{s}_{11}{ }^{\mathrm{E}}\left(10^{-12} \mathrm{~m}^{2} / \mathrm{N}\right)^{\mathrm{a}}$ & 45.4 & 24.8 & 16.4 & 12.8 & 7.7 \\
\hline Remanent polarization $\left(\mathrm{C} / \mathrm{m}^{2}\right)^{\mathrm{a}}$ & 0.25 & 0.38 & 0.48 & 0.29 & 0.32 \\
\hline Stress-induced charge density, $\left(\mathrm{C} / \mathrm{m}^{2}\right)$ & $0.19 \pm 0.01$ & $0.32 \pm 0.02$ & $0.48 \pm 0.02$ & $0.15 \pm 0.02$ & $0.32 \pm 0.02$ \\
\hline
\end{tabular}

${ }^{\mathrm{a}}$ The tolerance of parameters does not exceed $\pm 5 \%$. 

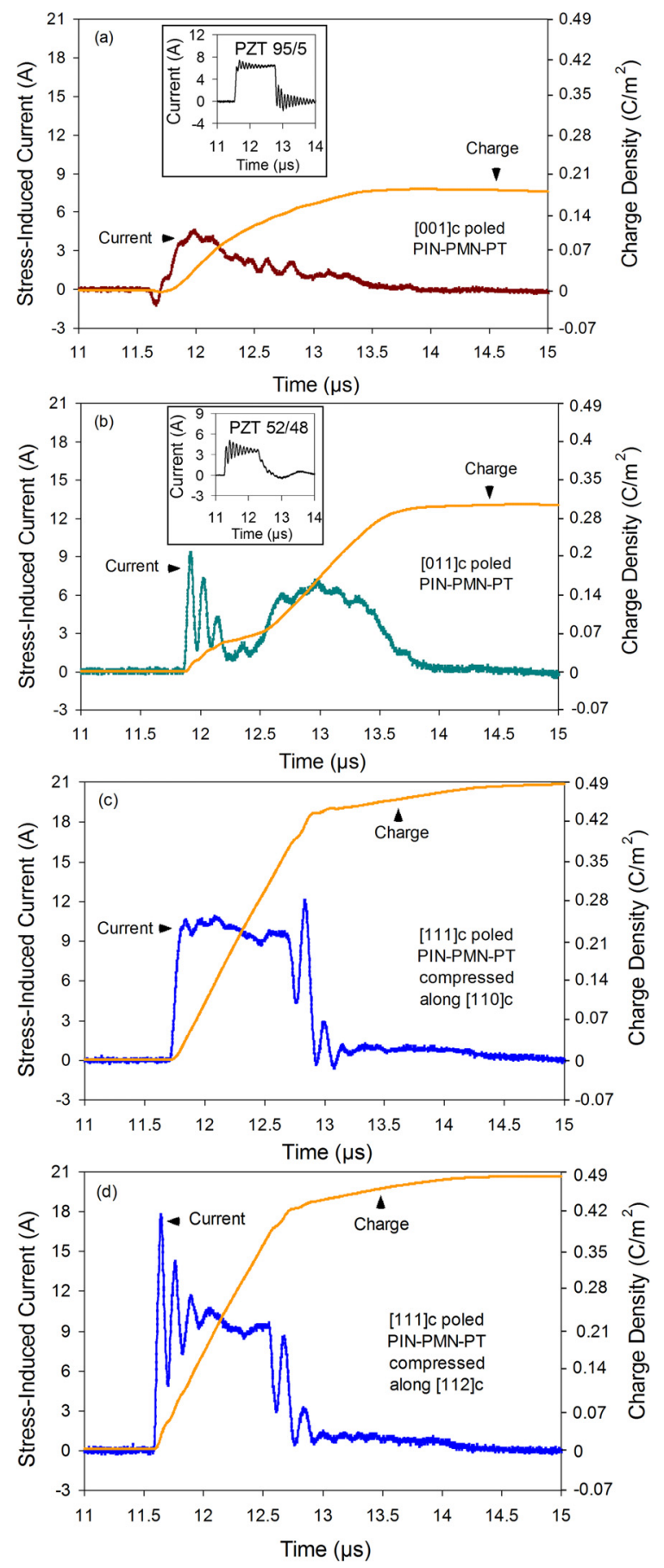

FIG. 1. Typical waveforms of stress-induced current and dynamics of electric charge released by rhombohedral PIN-PMN-PT multidomain and singledomain crystals: (a) multidomain crystal with $4 \mathrm{~mm}$ symmetry compressed along the [100]c direction; (b) multidomain crystal with $\mathrm{mm} 2$ symmetry compressed along the [100]c direction [the insets in (a) and (b) represent typical stress-induced current waveforms for PZT ceramic specimens used as reference objects in this study]; (c) and (d) single-domain crystals with $3 \mathrm{~m}$ symmetry compressed along the $[1 \overline{1} 0] \mathrm{c}$ and $[11 \overline{2}] \mathrm{c}$ directions, respectively.

complete depolarization of PZT 95/5 (see Table I) is the result of a stress-induced phase transformation from the ferroelectric (FE) rhombohedral to a non-polar anti-ferroelectric (AFE) orthorhombic phase. ${ }^{19-23}$ This transformation is also observed in hydrostatic studies ${ }^{14}$ where in PZT 95/5, the FE-to-AFE phase transformation occurs abruptly at a pressure of $0.32 \mathrm{GPa}$.

The uniaxial stress distribution in the ferroelectric crystals was determined by performing a simulation of the high strain rate loading using the CALE computer code (two-dimensional Arbitrary Lagrange/Eulerian second order accurate hydrodynamics program ${ }^{24}$ ). CALE results for the uniaxial stress distribution in the middle cross-section of the PIN-PMN-PT crystal are shown in Fig. SM-3 (supplementary material). The uniaxial stress is practically uniform in the cross-section, 3.9 $\pm 0.1 \mathrm{GPa}$. Based on these results, one can conclude that the complete depolarization of single-domain crystals with $3 m$ symmetry (Table I) cannot be explained by domain disappearance through a phase transformation to the higher symmetry non-polar cubic phase because the crystals do not undergo the rhombohedral-to-cubic phase transition under $3.9 \mathrm{GPa}$ uniaxial adiabatic compression. ${ }^{13}$

$\mathrm{X}$-ray diffraction (XRD) was performed on ferroelectric specimens before and after high strain rate loading (see supplementary material for description of the XRD measurements). Figure 2(a) shows the XRD patterns for PIN-PMN-PT crystals in the as-received condition. The dominant $\{001\}$, $\{011\}$, and $\{111\}$ reflections indicate that the crystal specimens were $[001] \mathrm{c},[011] \mathrm{c}$, and [111]c oriented in the rhombohedral phase. Figure 2(b) presents the XRD patterns for the [111]c oriented PIN-PMN-PT crystal and PZT ceramic specimens before loading. The XRD patterns indicate that the atomic structure of PIN-PMN-PT and PZT 95/5 specimens is very similar. The $\{111\}$ reflections show a predominantly rhombohedral distortion for PIN-PMN-PT and PZT 95/5. For PZT 52/48, the peak splitting that was observed in the $\{100\}$ and $\{200\}$ reflections indicates a predominantly tetragonal distortion.

Figure 2(c) shows XRD patterns for PIN-PMN-PT specimens before and after loading. The two XRD patterns have identical interplanar reflections. The $\{111\}$ reflections (see inset) indicate that the specimen was in the rhombohedral phase after loading. Similar results were observed for PZT 95/5 specimens. The X-ray diffraction results also indicate that PZT 95/5 depolarized by high strain rate loading is in the rhombohedral phase, i.e. it returns from the orthorhombic to the rhombohedral state after unloading. This observation is consistent with the results of hydrostatic studies of PZT 95/5. ${ }^{14}$ It was shown that when the pressure for hydrostatically loaded PZT $95 / 5$ is decreased from 0.32 to $0.14 \mathrm{GPa}$, it undergoes a transformation from the orthorhombic state back to the rhombohedral state.

Based on the obtained results, one could arrive at the conclusion that, similar to PZT 95/5, the PIN-PMN-PT crystals pass through a rhombohedral to non-polar orthorhombic (R-O) phase transition induced by uniaxial strain adiabatic compression, but if this were the case it should occur in all three types of domain engineered crystals. There is a well understood stress-induced $\mathrm{FE}_{\mathrm{R}}$ to $\mathrm{FE}_{\mathrm{O}}$ phase transformation occurring in the domain-engineered single crystals that can help to explain the observed depolarization behavior.

Complete depolarization of the [111]c oriented crystal cannot be explained by a shock-induced R-O phase transition because, unlike PZT 95/5, this is a polar-to-polar phase transformation. A possible explanation is provided by considering the multiaxial time-dependent state of stress in the crystals and its interaction with a known stress driven phase transformation. The [111]c oriented crystals transform from the singledomain rhombohedral to a lower symmetry multidomain orthorhombic phase, while the [011]c and [001]c oriented crystals transform from the multidomain rhombohedral to 

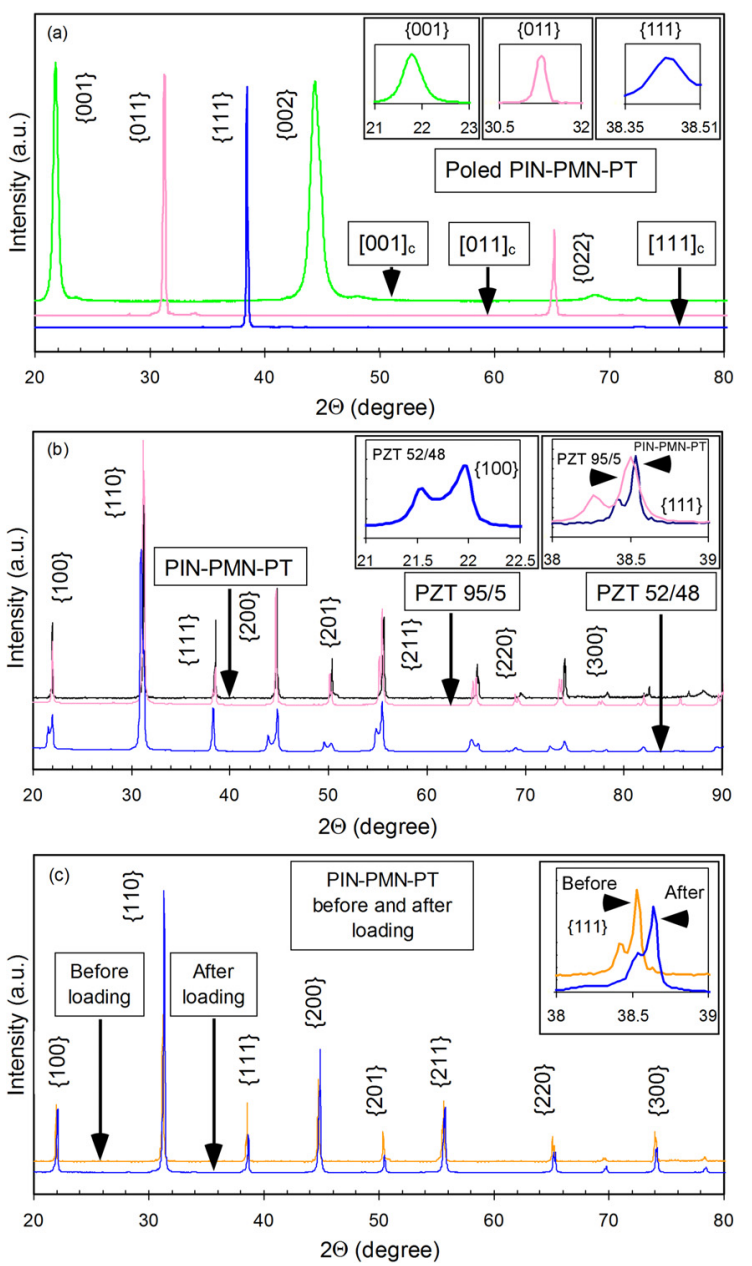

FIG. 2. XRD patterns for PIN-PMN-PT single crystals and PZT 95/5 and PZT 52/48 ceramic specimens in the as-received condition and after high strain rate loading experiments: (a) [001]c, [011]c, and [111]c oriented crystals in the as-received condition; (b) [111]c oriented crystal, PZT 95/5 and PZT 52/48 specimens in the as-received condition; (c) [111]c oriented crystals before and after high strain rate loading.

multidomain orthorhombic phase under certain states of stress. The stress-induced R-O phase transformation has been observed in [011]c and [001]c oriented PIN-PMN-PT crystals under quasi-static compressive stress not exceeding $100 \mathrm{MPa} .^{25,26}$ The R-O phase transformation in [011]c poled PIN-PMN-PT crystals associated with high cycle fatigue (polarization degradation) has also been observed. ${ }^{27}$

There is one more factor that can have an effect on depolarization of ferroelectrics under shock loading, i.e. the release waves traveling behind the shock front. They decompress the material in the directions perpendicular to the shock front propagation (see supplementary material for description of the release waves). The partial depolarization of transverse-shock-compressed PZT 52/48 (Table I) is the result of the effect of release waves. PZT 52/48 is predominantly in the tetragonal FE phase at ambient conditions, and it remains in the tetragonal FE phase under the experimental conditions used in this work. As the transverse shock propagates through the entire specimen, the effect of the release wave rotates part of the original polarization. ${ }^{18}$

Neither the polar-to-polar phase transformation in the [111]c oriented crystals nor the release wave effect makes the mechanism of complete depolarization of the crystals immediately apparent. However, the results described above give the grounds to propose a new mechanism responsible for complete stress-induced depolarization of the [111]c oriented crystals. This mechanism is based on a combination of the shock-induced phase transformation and reorientation of domains caused by release waves traveling behind the shock compressive front.

The PIN-PMN-PT crystal polarization variants in the rhombohedral and orthorhombic phases are shown in Fig. 3. As adiabatic compression is applied in the $Y$-direction, we propose a mechanism wherein a transformation from the rhombohedral to orthorhombic phase takes place in the crystals. The four variants of [001]c poled crystal being populated in the $R$ phase are transformed into four possible O-phase variants [Fig. 3(a)]. The two O-phase variants lying in the $X-Z$ plane become populated. The other two variants lying in the $Y-Z$ plane are eliminated due to the shock compression. There could be either a continuous rotation of the polarization through an intermediate monoclinic phase or a continuous evolution of the volume fraction of the $\mathrm{O}$ phase under shock compression.

There is no external electric field applied to the crystals along the $Z$-direction. Because of this, the two R-phase variants in the [011]c poled crystal are transformed into five Ophase variants [Fig. 3(b)]. The four of five O-phase variants having the $Y$-direction components are eliminated due to the

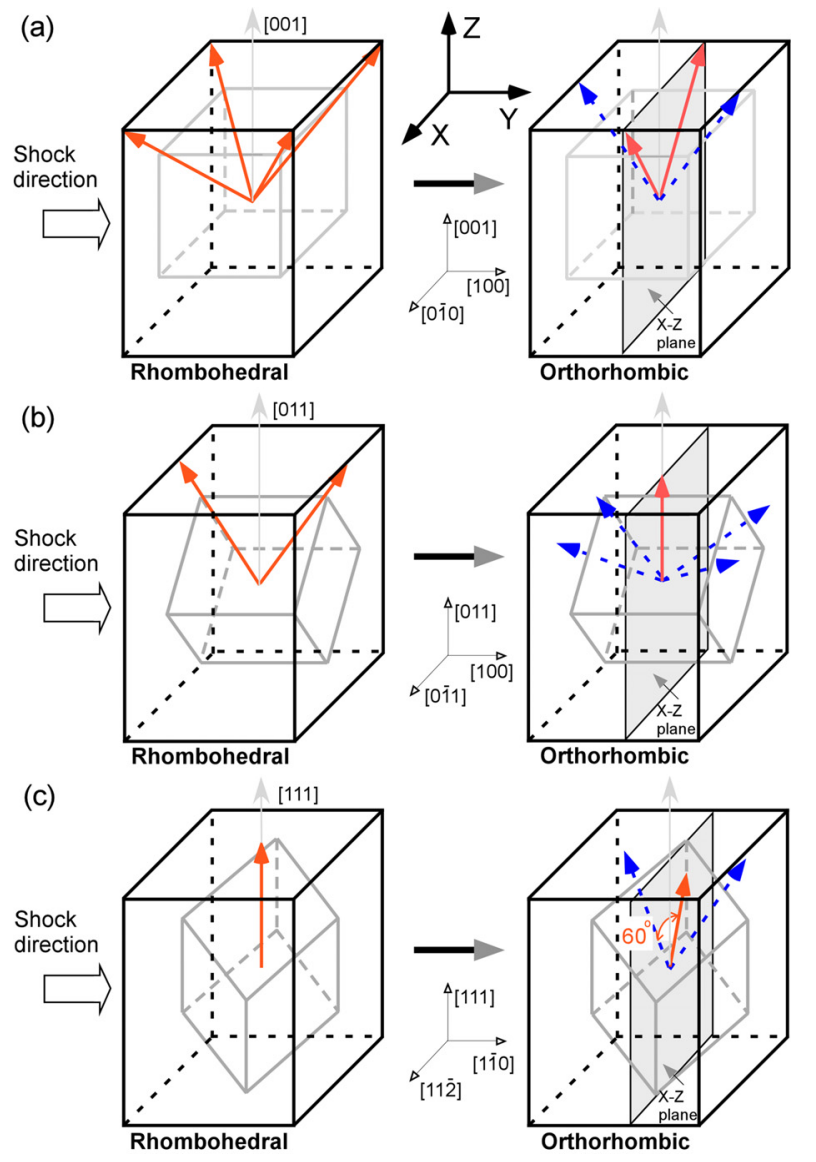

FIG. 3. Domain structures of PIN-PMN-PT single crystals and the rhombohedral-orthorhombic phase change process under high strain rate loading: (a) multidomain [001]c oriented crystal with $4 \mathrm{~mm}$ symmetry; (b) multidomain [011]c oriented crystal with $m m 2$ symmetry; (c) single-domain [111]c oriented crystal with $3 m$ symmetry. Cubic phase is shown by gray in each crystal cut. The orthorhombic phase variants eliminated due to the shock compression are marked by blue. 
shock compression and one variant becomes populated. The [001]c and [011]c crystals being in the orthorhombic phase are compressed in the $X-Z$ plane perpendicular to the shock wave direction by the release waves propagating behind the shock front. The time of mechanical destruction of the crystals behind the shock front is two orders of magnitude longer than the release wave propagation time. ${ }^{28}$ Upon passing the release waves, the crystal structure is transformed back into the rhombohedral phase with a different domain state and reduced polarization.

Opposite to the [001]c and [011]c poled crystals that undergo a multidomain-to-multidomain phase transition under shock compression, the polarization in the [111]c poled crystal begins in a single-domain rhombohedral state, which is no longer supported by the structure, and transforms to a multidomain orthorhombic state. There are no selection criteria (i.e. there is no priority in this process) in the multidomain state which is textured by the shock compression and release waves propagating behind the shock front. In the multidomain state, the polarization is not oriented in one direction as it was in the single-domain state. The one R-phase variant in the [111]c poled crystal is transformed into three O-phase variants [Fig. 3(c)]. The angle between the O-phase variants is $60^{\circ} .{ }^{29}$ The two of the three O-phase variants lying in the $Y-Z$ plane are eliminated due to the shock compression, and only one variant lying in the $X-Z$ plane becomes populated. The crystal has anisotropic elastic properties, resulting in a different release wave velocity perpendicular to the polarization than that parallel to the polarization direction making the stress pattern more complicated. The release wave provides a driving force for rotating the polarization in the $X-Z$ plane through the ferroelastic effect. A multidomain structure would develop to minimize the field, and therefore, the shocked crystal becomes completely depolarized when it is transformed back into the rhombohedral phase. There is one more important factor contributing to the complete depolarization of the single-domain crystals. In multidomain crystals, the domain walls provide a resistance to depolarization, and defects in the crystals can reinforce this through a memory effect. Both effects are expected to be greatly reduced in single-domain [111]c poled crystals. Based on all these, there is evidence that the mechanism of complete depolarization of the [111]c poled crystals is not related to a non-polar phase transition but is caused by a shock-induced transformation from the single-domain rhombohedral to multidomain orthorhombic phase compressed by the release waves leading to domain wall motion, polarization reorientation, and loss of the initial remanent polarization. To minimize energy, the resulting multidomain state is reoriented to give net zero polarization.

The mechanisms of stress-induced depolarization are similar for PIN-PMN-PT crystals with different domain configurations. However, the multidomain engineered PIN-PMNPT crystals are more resistant to stress-induced depolarization and are not completely depolarized under high stress. The differences in the depolarization of [001]c and [011]c poled crystals could be caused by different phase change processes and different domain wall motion and domain reorientation induced by complex time-dependent multi-directional stress.

A single-domain state makes the behavior of the crystals under high stress different from that of the multidomain crystals. The complete depolarization of single-domain [111]c poled crystals with high stress-induced electric charge density is very important for applications in ultrahigh-power transducers harvesting the ultimate energy density from ferroelectric materials. The mechanism of complete depolarization without transition to a non-polar phase proposed in this paper opens the way for development of a new class of high energy density ferroelectric materials for ultrahigh-power applications. It is possible that this mechanism provides complete depolarization for not only rhombohedral crystals but also single-domain crystals based on other structures.

See supplementary material for the polarization hysteresis loops of PIN-PMN-PT crystals, the description of the experimental setup used for high strain rate loading, the results of simulation of the uniaxial stress distribution in the crystals, the description of the release waves, and the description of the XRD measurements.

S.Z. acknowledge the support of ONRG (N62909-16-12126).

${ }^{1}$ S. E. Park and T. R. Shrout, J. Appl. Phys. 82(4), 1804 (1997).

${ }^{2}$ S. Zhang and F. Li, J. Appl. Phys. 111, 031301 (2012).

${ }^{3}$ H. Luo, G. Xu, H. Xu, P. Wang, and W. Yin, Jpn. J. Appl. Phys., Part 1 39, 5581 (2000).

${ }^{4}$ Y. Guo, H. Luo, T. He, and Z. Yin, Solid State Commun. 123, 417 (2002).

${ }^{5}$ G. Xu, K. Chen, D. Yang, and J. Li, Appl. Phys. Lett. 90, 032901 (2007).

${ }^{6}$ J. Tian, P. Han, X. Huang, and H. Pan, Appl. Phys. Lett. 91, 222903 (2007).

${ }^{7}$ S. Zhang, L. Jun, W. Hackenberger, and T. R. Shrout, J. Appl. Phys. 104, 064106 (2008).

${ }^{8}$ E. Sun and W. Cao, Prog. Mater. Sci. 65, 124 (2014).

${ }^{9}$ S. Zhang, F. Li, X. Jiang, J. Kim, J. Luo, and X. Geng, Prog. Mater. Sci. 68, 1 (2015).

${ }^{10}$ L. L. Altgilbers, J. Baird, B. Freeman, C. S. Lynch, and S. I. Shkuratov, Explosive Pulsed Power (Imperial College Press, London, UK, 2010).

${ }^{11}$ S. I. Shkuratov, J. Baird, and E. F. Talantsev, Appl. Phys. Lett. 102, 052906 (2013).

${ }^{12}$ B. Jaffe, W. R. Cook, Jr., and H. Jaffe, Piezoelectric Ceramics (Academic Press, London, New York, 1971).

${ }^{13}$ M. Ahart, S. Sinogeikin, O. Shebanova, D. Ikuta, Z.-G. Ye, H.-K. Mao, R. E. Cohen, and R. J. Hemley, Phys. Rev. B 86, 224111 (2012).

${ }^{14}$ I. J. Fritz, J. Appl. Phys. 49(9), 4922 (1978).

${ }^{15} \mathrm{~S}$. Zhang, F. Li, J. Luo, R. Xia, W. Hackenberger, and T. Shrout, Appl. Phys. Lett. 97, 132903 (2010).

${ }^{16}$ S. Zhang, F. Li, N. Sherlock, J. Luo, H. Lee, R. Xia, R. Meyer, W. Hackenberger, and T. Shrout, J. Cryst. Growth 318, 846 (2011).

${ }^{17}$ X. Huo, S. Zhang, G. Liu, R. Zhang, J. Luo, R. Sahul, W. Cao, and T. Shrout, J. Appl. Phys. 113, 074106 (2013).

${ }^{18}$ S. I. Shkuratov, J. Baird, V. G. Antipov, E. F. Talantsev, H. R. Jo, J. C. Valadez, and C. S. Lynch, Appl. Phys. Lett. 104, 212901 (2014).

${ }^{19}$ W. J. Halpin, J. Appl. Phys. 27(1), 153 (1966).

${ }^{20}$ D. G. Doran, J. Appl. Phys. 39(1), 40 (1968).

${ }^{21}$ R. E. Setchell, J. Appl. Phys. 97, 013507 (2005).

${ }^{22}$ R. E. Setchell, J. Appl. Phys. 94(1), 573 (2003).

${ }^{23}$ R. E. Setchell, J. Appl. Phys. 101, 053525 (2007).

${ }^{24}$ E. E. Tipton, in Proceedings of the Fourth International Conference on Megagauss Magnetic Field Generation and Related Topics, edited by C. M. Fowler, R. S. Caird, and D. J. Erickson (Plenum Press, New York, 1987), p. 299.

${ }^{25}$ S. Zhang, S. Taylor, F. Li, J. Luo, and R. J. Meyer, Jr., Appl. Phys. Lett. 102, 172902 (2013).

${ }^{26}$ J. A. Gallagher, J. Tian, and C. S. Lynch, Appl. Phys. Lett. 105, 052909 (2014).

${ }^{27}$ S. Zhang, J. Luo, F. Li, and R. J. Meyer, Acta Mater. 58, 3773 (2010).

${ }^{28}$ S. I. Shkuratov, S. I. E. F. Talantsev, and J. Baird, J. Appl. Phys. 110, 024113 (2011).

${ }^{29}$ M. Davis, Master Degree thesis No. 3513, Ecole Polytechnique Federale De Lausanne, 2006. 